\title{
Un projet de révision de loi relative à l'exercice du droit syndical
}

\section{Chakib Boukli Hacène}

\section{(2) OpenEdition}

1 Journals

\section{Édition électronique}

URL : https://journals.openedition.org/rdctss/1247

DOI : $10.4000 /$ rdctss. 1247

ISSN : 2262-9815

Éditeur

Centre de droit comparé du travail et de la sécurité sociale

\section{Édition imprimée}

Date de publication : 1 avril 2020

Pagination : 144-147

ISSN : 2117-4350

\section{Référence électronique}

Chakib Boukli Hacène, « Un projet de révision de loi relative à l'exercice du droit syndical », Revue de droit comparé du travail et de la sécurité sociale [En ligne], 1 | 2020, mis en ligne le 01 novembre 2021, consulté le 11 novembre 2021. URL : http://journals.openedition.org/rdctss/1247 ; DOI : https:// doi.org/10.4000/rdctss. 1247

\section{(c) $(7)(9$}

Revue de droit comparé du travail et de la sécurité sociale est mise à disposition selon les termes de la Licence Creative Commons Attribution - Pas d'Utilisation Commerciale - Pas de Modification 4.0 International. 


\title{
CHAKIB BOUKLI HACÈNE
}

\author{
UNIVERSITÉ DE SAÏDA
}

\section{UN PROJET DE RÉVISION DE LOI RELATIVE À L'EXERCICE DU DROIT SYNDICAL}

Durant ces derniers mois, l'Algérie a connu des manifestations populaires et pacifiques réclamant davantage de démocratie et plus de liberté. Sous cette pression populaire, le gouvernement de transition (dit de gestion des affaires courantes) s'est montré conciliant vis-à-vis des syndicats autonomes, après des décennies d'injustice et de persécution sous l'ancien régime. II a ainsi pris différentes mesures visant à permettre l'enregistrement d'un nombre important d'organisations syndicales, dont la demande en ce sens était en souffrance, parfois depuis plusieurs années.

Le gouvernement a également engagé plusieurs réformes juridiques, notamment celle relative à l'exercice du droit syndical, en vue d'une plus grande adaptation aux normes internationales. Initiée par les autorités publiques et actuellement au stade d'avant-projet de loi, cette réforme se base essentiellement sur les conclusions du Rapport de la Commission d'experts pour l'application des Conventions et Recommandations, présenté lors de la 108 session de la Conférence Internationale du Travail, du 10 au 21 juin 2019 à Genève.

Après avoir analysé les Recommandations de la Commission de l'application des normes de la Conférence Internationale du Travail (I), la présente contribution examinera la réponse apportée par le gouvernement algérien dans le cadre de l'avant-projet de loi modifiant et complétant la loi du 2 juin 1990 relative aux modalités d'exercice du droit syndical (II).

\section{I - LES RECOMMANDATIONS DE LA COMMISSION DE L'APPLICATION DES NORMES}

Selon le rapport susmentionné, les griefs formulés par la Commission à l'encontre du gouvernement algérien remontent à 2011 et se résument en deux points essentiels.

Sur un plan législatif : à plusieurs reprises, la Commission a recommandé au gouvernement algérien de mettre sa législation du travail - notamment la loi $n^{\circ}$ 90-14 du 2 juin 1990 relative aux modalités d'exercice du droit syndical - en conformité avec la Convention internationale du travail $n^{\circ} 87$. La Commission reprochait en effet le contenu de l'article 6 de cette loi qui, contrairement à l'article 2 de la Convention $n^{\circ} 87$, limitait le droit de constituer une organisation syndicale aux seules personnes de nationalité algérienne d'origine ou acquise depuis au moins dix ans. Elle appelait donc à une révision rapide dudit article, visant à supprimer l'exigence de nationalité et à reconnaître à tous les travailleurs, sans aucune distinction de nationalité, le droit de constituer une organisation syndicale. Par ailleurs, la Commission demandait depuis de nombreuses années au gouvernement algérien de procéder sans délai à la révision de l'article 4 de la loi n90-14 du 2 juin 1990 afin, conformément à l'article 5 de la Convention n87, de lever tous les obstacles à la création de fédérations et confédérations par les organisations de travailleurs, quel que soit le secteur auquel ils appartiennent. En effet, la loi algérienne exige pour 
la constitution des unions, fédérations et confédérations syndicales l'appartenance de ces dernières à la même profession, branche ou secteur d'activité. L'objectif consistait à empêcher la constitution de confédérations nationales regroupant des syndicats de divers secteurs d'activité ou branches professionnelles. C'est sur la base de cet argument que les autorités algériennes ont ainsi reconnu I'Union Générale des Travailleurs Algériens (UGTA) comme étant l'unique organisation syndicale pouvant exercer au niveau national, toutes branches d'activités confondues ${ }^{1}$.

Sur un deuxième plan d'ordre pratique et relatif à l'enregistrement des syndicats, la Commission a constaté, depuis de nombreuses années, une certaine réticence de la part des autorités algériennes à enregistrer les organisations syndicales dites autonomes. Constatant des délais particulièrement longs et non justifiés - parfois de plusieurs années dans le traitement des demandes d'enregistrement, alors même que la loi algérienne fixe un délai de 30 jours maximum pour la délivrance du récépissé d'enregistrement, la Commission a ainsi rappelé au gouvernement algérien le fait que la Convention $n^{\circ} 87$ ne subordonne nullement la constitution d'un syndicat à une autorisation administrative préalable et n'accorde pas, de ce fait, aux autorités publiques des pays signataires un pouvoir discrétionnaire pour refuser la constitution d'un syndicat. En conséquence, la Commission a exhorté le gouvernement à respecter la liberté de constitution des syndicats de travailleurs, telle que prévue à la fois dans la Convention n87 mais également par la loi algérienne.

\section{II - LA RÉPONSE DU GOUVERNEMENT ALGÉRIEN}

Une fois n'est pas coutume, les Recommandations de la Commission de l'application des normes de la Conférence Internationale du Travail semblent trouver un écho favorable et diligent auprès du gouvernement transitoire algérien, dont la mission a pris fin le 12 décembre dernier. En effet, il a pris des mesures concrètes envers les syndicats, proposant de modifier les articles 4, 6, 8, 9,56 et 59 de la loi relative aux modalités d'exercice du droit syndical de 1990, dans le cadre de deux avant-projets de loi. Il faut signaler que cette démarche contraste fortement avec les réformes engagées par les pouvoirs publics algériens en matière de droit du travail dans ses relations individuelles et collectives, qui s'illustrent notamment dans le contenu du nouveau Code du travail. Ainsi le Gouvernement a-t-il adopté deux avant-projets de loi : le premier en 2015 sous la forme d'un Code du travail régissant toutes les relations individuelles et collectives de travail, y compris le droit syndical ; et le second paru en septembre 2019, modifiant et complétant la loi du 2 juin 1990 relative aux modalités d'exercice du droit syndical, étudié plus en détails ci-dessous.

S'agissant du contenu de ce projet de loi, la lecture de son exposé des motifs laisse à penser que le texte n'a été élaboré que dans le seul but de fournir une réponse aux griefs formulés à l'Algérie, par la Commission d'experts et le Comité de la liberté syndicale de I'OIT, pour non-respect de la Convention n87. C'est dans ce sens que l'avant-projet de loi a introduit un amendement aux dispositions de la loi de 1990 sur l'exercice du droit syndical, permettant la constitution de fédérations, d'unions et de confédérations syndicales, nonobstant la condition de similitude de profession, de branche ou secteur

1 L'article 11 de la loi n 90-14 du 2 juin 1990 relative aux modalités d'exercice du droit syndical dispense les organisations syndicales légalement constituées à la date de la promulgation de la loi (comme I'UGTA) de la déclaration de constitution. 
d'activité. II convient également de souligner que ce nouveau texte retient une définition surprenante de la fédération, de l'union et de la confédération, en se basant sur un critère purement numérique : la fédération est ainsi considérée comme moins importante en nombre d'adhérents que l'union ou la confédération - mises pour leur part sur un pied d'égalité - contrairement à la législation actuelle qui estime l'union plus petite en nombre d'adhérents que les deux autres.

En outre, le projet de loi propose de supprimer la condition de nationalité afin de permettre aux travailleurs et aux employeurs étrangers de constituer des organisations syndicales, et d'accéder éventuellement aux fonctions d'administration ou de direction. Cet amendement semble n'exister que pour satisfaire aux exigences exprimées par les instances du Bureau International du Travail (BIT) car, dans la pratique, il reste difficile pour un travailleur étranger de fonder un syndicat, eu égard aux conditions contraignantes de son employabilité sur le marché du travail algérien.

Concernant l'enregistrement problématique des organisations syndicales, l'avant-projet de loi avance une proposition singulière dans la mesure où il suggère de répondre à la nécessité d'assouplir les formalités de publicité auxquelles l'organisation syndicale est soumise lors de sa constitution, par la suppression de la seule mention « dans au moins un quotidien national d'information ». Rappelons en effet qu'il est fait obligation aux organisations syndicales, dans l'actuelle législation du travail, d'accomplir à leur frais des formalités de publicité dans au moins un quotidien national d'information. C'est une procédure simple qui ne recèle aucune difficulté particulière. En réalité, cet amendement constitue « une fuite en avant » de la part des autorités algériennes puisque les organisations syndicales dites autonomes n'ont, à aucun moment, protesté envers les formalités de publicité.

En revanche, le réel problème réside dans la délivrance du récépissé d'enregistrement suite au dépôt de la déclaration de constitution. Dès lors, la solution consisterait davantage à déterminer et à clarifier le rôle des autorités publiques compétentes ${ }^{2}$ dans la délivrance dudit récépissé d'enregistrement, plutôt que d'amender l'alinéa relatif à la formalité de publicité. Néanmoins, il ne s'agit nullement de doter les autorités compétentes d'un pouvoir discrétionnaire dans la mesure où, in fine, il reviendra au juge de décider de la conformité du dossier de constitution de l'organisation syndicale au regard de la loi en vigueur.

Enfin, cet avant-projet de loi vise à renforcer la protection apportée au délégué syndical contre tout licenciement intervenu en violation de la procédure légale, en le qualifiant de nul et de nul effet. Ainsi, en cas de refus manifeste de l'employeur de procéder à la réintégration du délégué syndical, l'inspecteur du travail territorialement compétent dresse un procès-verbal (ci-après PV) « de constat » qu'il remet au travailleur victime du licenciement illégal, afin de lui permettre de l'utiliser dans son action en réintégration devant la juridiction sociale compétente. Sur ce point, il semble que les rédacteurs de l'avant-projet, partant de l'intention de rendre la protection des délégués syndicaux plus opérante et plus efficace, l'ont au final davantage compliquée. Tout d'abord, l'avant-projet de loi introduit ce nouveau PV « de constat » en sus des différentes observations écrites, mises en demeure,

2 Le Wali (Préfet) ou le Ministre chargé du travail. 


\section{AlgÉRIE}

procès-verbaux d'infraction et de conciliation ${ }^{3}$ que l'inspecteur du travail est déjà habilité à établir dans l'actuelle législation.

Par ailleurs, la loi actuelle offre dès à présent la possibilité à l'inspecteur du travail, en pareilles circonstances, de dresser un procès-verbal d'infraction et de saisir directement la juridiction compétente ; en l'occurrence la juridiction du référé qui peut exceptionnellement statuer sur la réintégration du délégué licencié, par décision exécutoire dans un délai n'excédant pas 60 jours.

Pour conclure, force est de constater que cet avant-projet de loi concernant l'exercice du droit syndical constitue un travail peu satisfaisant, engagé dans une relative précipitation, sans réflexion ni concertation préalables. De plus, avec l'installation du nouveau gouvernement algérien, nul ne sait si le nouveau Ministre du travail poursuivra l'élaboration de cet avant-projet de loi - étant donné qu'il est le fruit du travail de son prédécesseur - en le présentant au Conseil des Ministres puis au vote du Parlement. II se pourrait également qu'il lui préfère l'avant-projet de loi de 2015, qui élabore un Code du travail global.

A cet égard, signalons que la nouvelle Constitution algérienne en cours de préparation va probablement impacter de nombreux textes de loi actuels relatifs au droit du travail, au risque que ces derniers ne deviennent anticonstitutionnels. II est à craindre que cette " révision générale » à venir incite le Ministre du travail à apporter sa pierre à l'édifice, notamment en proposant son nouveau projet de loi relatif au droit du travail.

3 Voir M. N.-E. Koriche, " Droit du travail : Les transformations du droit du travail entre statut et contrat, relation d'emploi et de travail, une contractualisation relative ", Office des publications universitaires, Alger, 2009, p. 65. 